\title{
Mechanical and Electric Properties of Ultra-High-Molecular Weight Polyethylene and Carbon Black Particle Blends
}

\author{
Chunye Xu, Yasuyuki Agari, ${ }^{*}$ and Masaru Matsuo ${ }^{\dagger}$ \\ Department of Textile and Apparel Science, Faculty of Human Life and Environment, \\ Nara Women's University, Nara 630-8506, Japan \\ * Osaka Municipal Technical Research Institute, Osaka 536-8553, Japan
}

(Received July 3, 1997)

\begin{abstract}
Composite materials of ultra-high-molecular weight polyethylene (UHMWPE)-carbon black (CB) particles were prepared by gelation/crystallization from solution in order to obtain the composite materials with various electric conductivities. UHMWPE-CB compositions chosen were 1/0.25, 1/0.5, 1/0.75, 1/1, 1/3, 1/5, and 1/9. Electric conductivity increased with increasing $\mathrm{CB}$ content and was in the range of $10^{-9}$ to $1 \Omega^{-1} \mathrm{~cm}^{-1}$ corresponding to semiconductors. These values were almost independent of temperature up to $175^{\circ} \mathrm{C}$ indicating thermal stability. Elongation was carried out in a hot oven at $135^{\circ} \mathrm{C}$. Drawability was less pronounced as $\mathrm{CB}$ content increased. For example, the maximum achievable draw ratio of $1 / 1$ composition ( $50 \%$ CB content) was 70 -fold, while that of 1/0.25 (20\% CB content), 150-fold. The corresponding Young's modulus and tensile strength for the $1 / 0.25$ blend were 73.6 and $1.31 \mathrm{GPa}$, respectively, while those for the $1 / 1$ blend were 25.4 and $0.46 \mathrm{GPa}$, respectively. The materials with $\mathrm{CB}>83 \%$ could not be elongated in spite of good impact resistance. The electric conductivity for the drawn blends was anisotropic. The values in the stretching direction decreased with increasing draw ratio, while the values in the thickness direction increased slightly.

KEY WORDS Composite Materials / Ultra-High-Molecular Weight Polyethylene / Carbon Black / Electric Conductivity / Thermal Stability / Drawability /
\end{abstract}

Until quite recently, most polymers were limited to specialized uses in products such as fibers, films, and coatings or to their familiar role as light-duty, inexpensive plastic materials. Polymers in the pure state are excellent electrical insulators. But polymers can be modified in processing to be relatively good electrical conductors either by doping with impurities or by simply creating mixtures of a good conductor and a polymer. ${ }^{1-26}$ Accordingly, polymeric materials should be prepared with relatively higher conductivity than is currently available. At present, the later category is more useful than the former because of the drastic oxygenation of conductive polymers and a number of papers have been reported in accordance with this guide-line.

Among conductor-filled polymers, carbon black (CB) filled polyethylene (PE) is one of typical good conductive materials. Considerable experimental efforts have been devoted over several decades to electrical studies of the basic phenomenon. The focus was concentrated on positive temperature coefficient (PTC) effect associated with a drastic decrease in electric conductivity at temperature range close to the melting point of $\mathrm{PE}$ and interesting results have been reported in terms of theoretical and experimental aspects. ${ }^{3-11,18-20}$

Apart from the above concept, it is of interest to consider the preparation of $\mathrm{CB}$ filled $\mathrm{PE}$ with thermal stability and with various CB content up to $90 \%$ in terms of various industrial utilities. For dimensional stability at elevated temperature, low-molecular weight polyethylene (LMWPE), molecular weight less than $5 \times 10^{5}$, is not suitable because of a high melt index. Accordingly, ultra-high-molecular weight polyethylene (UHMWPE), molecular weight higher than $10^{6}$, was used as a test specimen, since the shape of UHMWPE films could be maintained without any flowability for several hours

\footnotetext{
† To whom all correspondence should be addressed.
}

even at $180^{\circ} \mathrm{C}$ higher than the equilibrium melting point. $^{27}$ Such high viscosity, however, invokes the difficulty in preparing composite materials by kneading. In spite of a lot of trials for introducing CB particles under kneading, a uniform mixing of UHMWPE powder and $\mathrm{CB}$ particles has never been successful and the kneading method is limited to LMWPE-CB blend systems.

To avoid such difficulty, this paper deals with a special sample preparation by the introduction of large amounts of CB particles into UHMWPE solutions and subsequently by the gelation/crystallization from the solutions. ${ }^{28-33}$ This method has advantage for preparing UHMWPE-CB blended systems with $90 \%$ CB content and electric conductivities of the various composite materials were in the wide range from $10^{-9}$ to $1 \Omega^{-1} \mathrm{~cm}^{-1}$ corresponding to semiconductors. This interesting phenomenon was mainly investigated morphologically using wide angle X-ray diffraction (WAXD), small-angle $\mathrm{X}$-ray scattering (SAXS) and scanning electron microscopy.

\section{EXPERIMENTAL}

The sample preparation was done using linear polyethylene with the average molecular weight of $6 \times 10^{6}$ and two kinds of CB particles, low structure (L.S.) CB with commercial BLACK PEARLS 880 and high structure (H.S.) CB with commercial BALKAN XC-72. Observation by electron microscopy and DBP measurements indicated particle's size of a L.S. CB particle to be about $16 \mathrm{~nm}$ and the number of connected particles indicating a beads-like structure, about 10, while that of a H.S. CB particles, about $30 \mathrm{~nm}$ and the number, about 17. The two kinds of the CB particles were furnished by Cabot Co., Ltd. The naming of L.S. CB and H.S. CB was done to distinguish the two kinds of $\mathrm{CB}$ particles, 
since the beads-like structure length of BLACK PEARLS 880 is shorter than that of BALKAN XC-72 but BLACK PEARLS 880 is much longer than commercial $C B$ particles termed as low structure L.S. CB usually. The solvent of UHMWPE was decalin. UHMWPE-CB compositions were $1 / 0.25,1 / 0.5,1 / 0.75,1 / 1,1 / 3,1 / 5$, and $1 / 9$ by a weight fraction. Solutions containing UHMWPE $(0.5 \% \mathrm{w} / \mathrm{w})$ and $\mathrm{CB}$ particles were prepared by heating the well-blended polymer/solvent mixture at $150^{\circ} \mathrm{C}$ for 30 min under nitrogen ${ }^{31-33}$ to ensure uniform dispersion of $\mathrm{CB}$ particles. The volume of the solutions was controlled to obtain the film whose thickness is about $700 \mu \mathrm{m}$. The hot homogenized solution was quenched by pouring it into an aluminum tray at room temperature, thus generating a gel. The decalin was allowed to evaporate from the gel under ambient conditions. The nearly dried films were vacuum-dried for 3 days at $50^{\circ} \mathrm{C}$ to remove residual trace of decalin as much as possible. Decalin within the films could not be observed from FT-IR spectra.

The composite material was cut into strips of length $30 \mathrm{~mm}$ and width $10 \mathrm{~mm}$. The strip was clamped in a manual stretching device in such a way that the length to be drawn was $20 \mathrm{~mm}$. The specimens were stretched in a hot oven at $135^{\circ} \mathrm{C}$.

The densities of the PE dry gel and the melt films were measured by a pycnometer using a mixture of carbon tetrachloride and toluene as a medium. Before the measurement was made, the specimen was cut into fragments and vacuum-dried for 1 day. Crystallinity was calculated assuming intrinsic densities of the crystalline and amorphous phases to be 1.000 and $0.852 \mathrm{~g} \mathrm{~cm}^{-3}$, respectively. ${ }^{34}$

The thermal behavior was estimated from the melting endotherm on differential scanning calorimetry (DSC) curves. The sample was placed in a standard aluminum sample pan and heated at a constant rate of $10^{\circ} \mathrm{C} \mathrm{min}{ }^{-1}$.

Scanning electron micrographs were obtained with a JSM-T300.

$\mathrm{X}$-Ray measurements were carried out with a $12-\mathrm{kW}$ rotating-anode $\mathrm{X}$-ray generator (Rigaku RAD-rA). WAXD patterns were obtained with a flat camera using $\mathrm{Cu}-K_{\alpha}$ radiation at $200 \mathrm{~mA}$ and $40 \mathrm{kV}$. SAXS patterns were obtained with a flat-film camera. The exposure time was about $48 \mathrm{~h}$ by point focus with a three-pin hole collimator system.

The complex dynamic tensile modulus was measured at $10 \mathrm{~Hz}$ over the temperature from -150 to $300^{\circ} \mathrm{C}$ by using a viscoelastic spectrometer (VES-F) obtained from Iwamoto Machine Co., Ltd. The length of the specimen between the jaws was $40 \mathrm{~mm}$ and width, about $1.5 \mathrm{~mm}$. During measurement, the film was subjected to be a static tensile strain to place the sample in tension during the axial sinusoidal oscillation which had a peak deformation of $0.05-0.1 \% .^{34}$ The complex dynamic modulus was measured by imposing a small dynamic strain to assure linear viscoelastic behavior of the specimens.

Electric conductivity was measured by the two-terminal method for stretching direction (direction parallel to the film surface for undrawn films) and film thickness direction at $23 \pm 2{ }^{\circ} \mathrm{C}$ and at relative humidity of $50 \pm 5 \%$. To prepare the test specimens, the composite material was cut into strips of length $25 \mathrm{~mm}$ and width

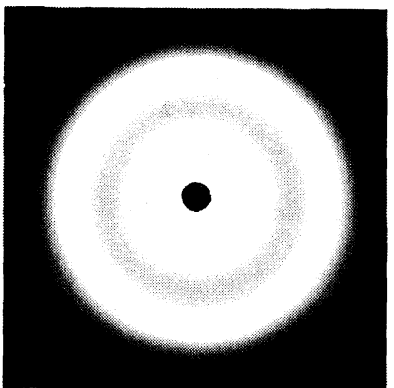

(a) L.S.

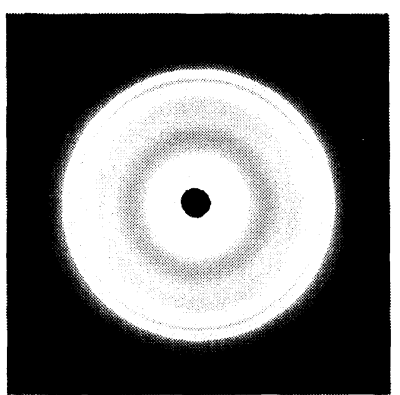

(c) $1 / 1$ (L.S.)

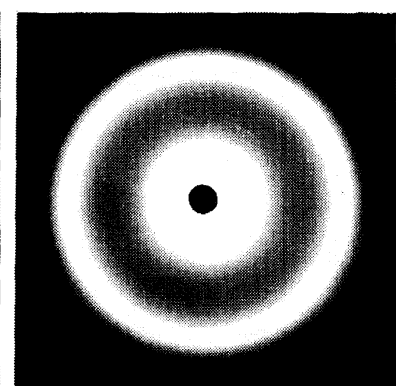

(b) H.S.

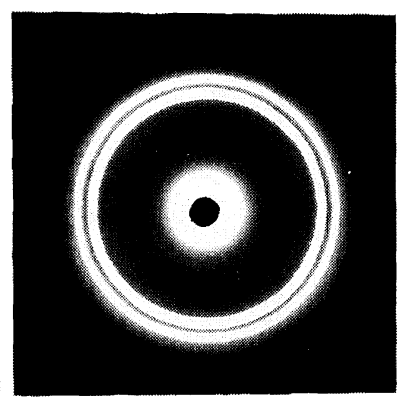

(d) $1 / 1$ (H.S.)
Figure 1. WAXD patterns: (a) L.S. CB particles; (b) H.S. CB particles; (c) UHMWPE-L.S. CB particles with $1 / 1$ composition; (d) UHMWPE-H.S. CB particles with $1 / 1$ composition.

$15 \mathrm{~mm}$. Before measurement, the specimen was polished by sandpaper to level off the both sides smoothly, since contact resistance depends on the sample surface quality. The conductivity $>10^{-6} \Omega^{-1} \mathrm{~cm}^{-1}$ was calculated by measuring voltage at constant current of $0.1-1 \mathrm{~mA}$. The conductivity $<10^{-6} \Omega^{-1} \mathrm{~cm}^{-1}$ was calculated by measuring current yielded at voltage of 10 VDC. The electric conductivity in the stretching direction was measured using the specimen clamped between copper metal jaws. For undrawn films, the stretching direction corresponded to the arbitrary direction on the film surface. The distance between jaws was $10 \mathrm{~mm}$. In the film thickness direction, electric conductivity was measured by sandwiching the material between two copper plates.

\section{RESULTS AND DISCUSSION}

\section{Undrawn Composite Materials}

Patterns (a) and (b) in Figure 1 show WAXD patterns from L.S. and H.S. CB particles, respectively and patterns (c) and (d), UHMWPE-L.S. CB particles with $1 / 1$ composition and UHMWPE-H.S. CB particles with $1 / 1$ composition, respectively. The broad halo is observed for patterns (a) and (b). The air scattering from L.S. CB particles appeared at the lower scattering angle side is more intense than that from H.S. CB particles. In patterns (c) and (d), the strong diffuse halo overlapped the diffraction rings of the (110) and (200) planes of PE crystallites. The pattern of the composite material containing L.S. CB particles shows more indistinct diffraction rings of the (110) and (200) planes in comparison with that containing H.S. CB particles. To analyze the complicated X-ray profile of the composite materials clearly, the diffraction intensity distributions were observed for the two blends.

Column (a) in Figure 2 shows the scattered intensity distribution against twice the Bragg angle $\left(\theta_{\mathrm{B}}\right)$ observed 


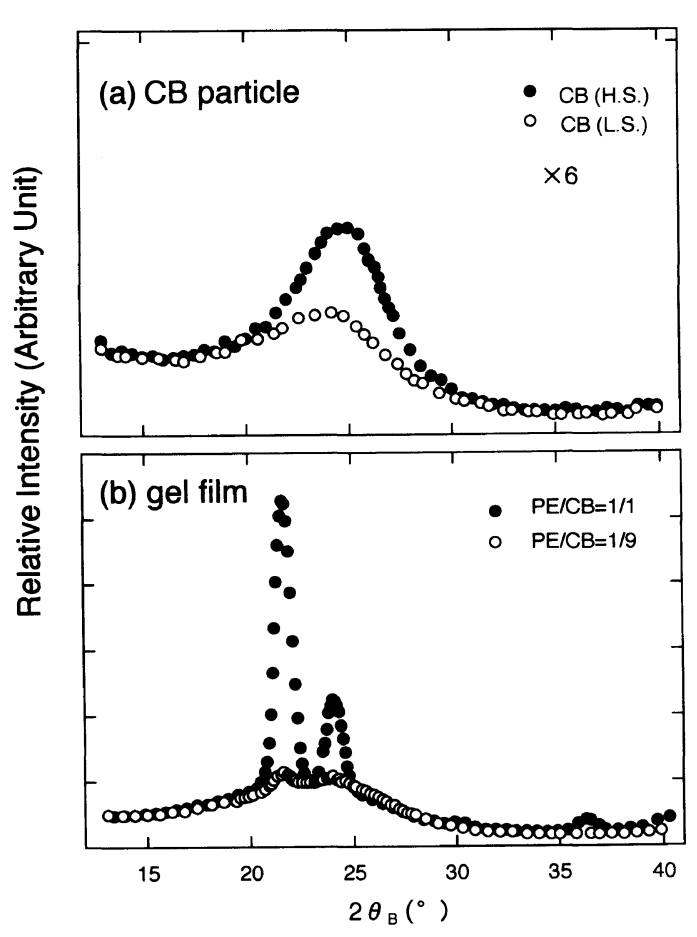

Figure 2. X-ray intensity distributions: (a) L.S. and H.S. CB particles; (b) the UHMWPE-L.S. CB with $1 / 1$ and $1 / 9$ compositions.
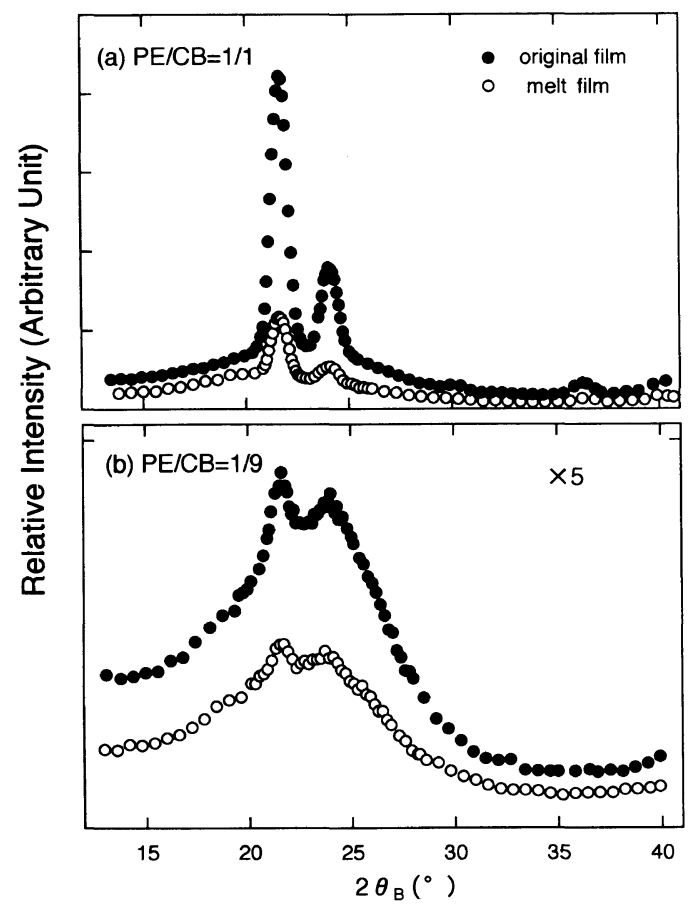

Figure 3. X-ray intensity distributions: (a) UHMWPE-L.S. CB blend films with 1/1 composition; (b) UHMWPE-L.S. CB blend films with $1 / 9$ compositions.

for the L.S. and H.S. CB particles and column (b), diffraction intensity distribution for the PE-L.S. CB blends observed by step-scanning method. The plots were obtained after correction for absorption, polarization, and air scattering. The diffuse scattering peak of the L.S. and H.S. CB particles appeared at 23.8 and $24.8^{\circ}$, respectively. The scattered intensity was measured for the same X-ray irradiated volume with regard to the two kinds of CB particles. The scattered intensity from the
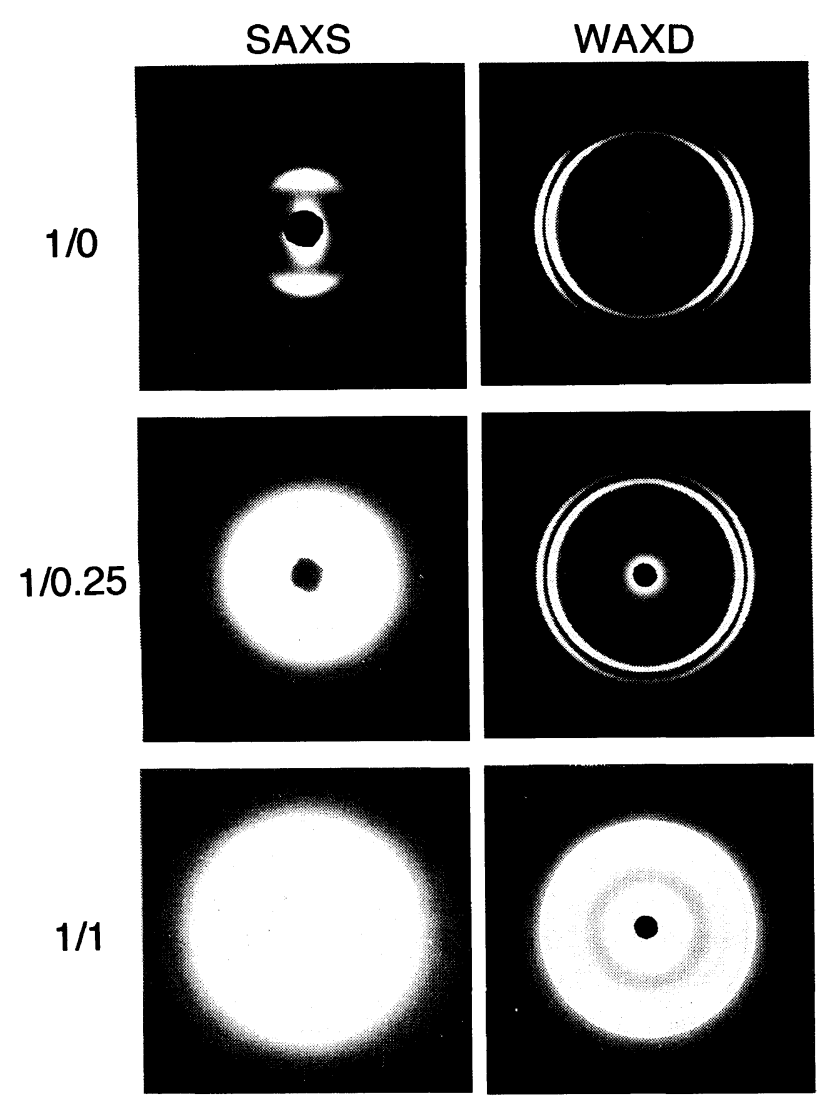

$1 / 9$
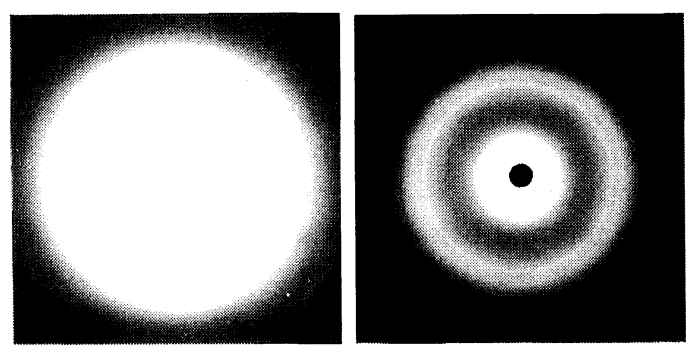

Figure 4. SAXS and WAXD patterns (end view) from blend materials with the indicated compositions.

H.S. CB particles is stronger than that from L.S. CB particles indicating that the chain arrangement within H.S. CB particles has better order. The intensity distribution curves for the $1 / 1$ and $1 / 9$ blends in column (b) were observed for L.S. CB particles, since the uniform dispersion of L.S. CB particles under the sample preparation is slightly easier in comparison with that of H.S. CB ones. In column (b), the diffraction intensities of the (110) and (200) reflections become weaker with increasing L.S. CB content. The peaks for the (110) and (200) reflection for the $1 / 1$ blend appeared at 21.6 and $24.0^{\circ}$, respectively, without any peak shift by the introduction of the particles.

Figure 3 shows diffraction intensity distributions for the original dry gel composite material and for the material (melt film) obtained by melting the original materials at $180^{\circ} \mathrm{C}$ for $22 \mathrm{~min}$ and recrystallizing them at room temperature. The experiment was carried out to check the relationship between crystallinity and electric conductivity, which shall be discussed later (see Figure 7). The upper and lower columns in Figure 3 correspond to the $1 / 1$ and $1 / 9$ blends, respectively. As shown in column (a), a drastic decrease in peak intensity was 


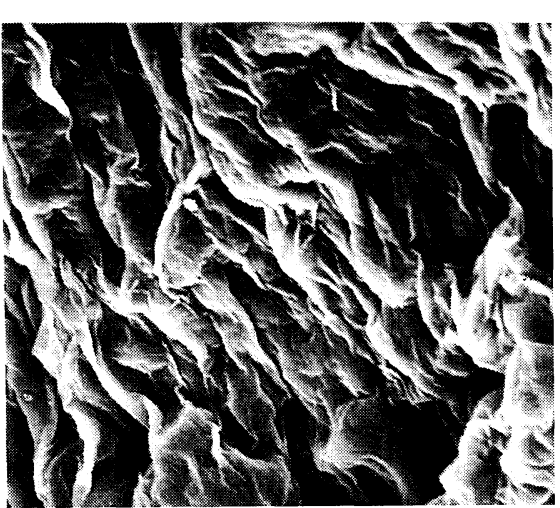

(a) $\mathrm{PE} / \mathrm{CB}=1 / 1$

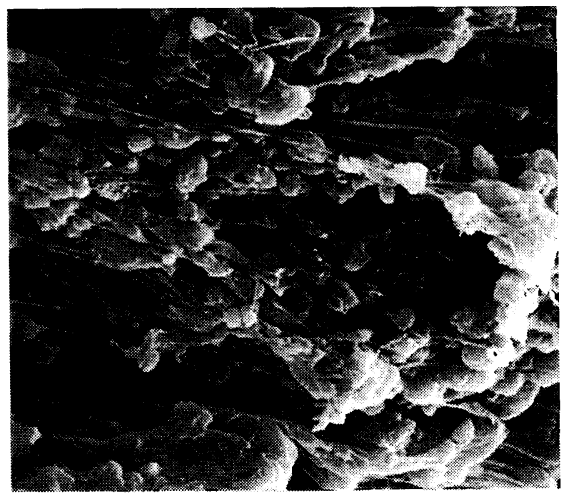

(c) $\mathrm{PE} / \mathrm{CB}=1 / 9$

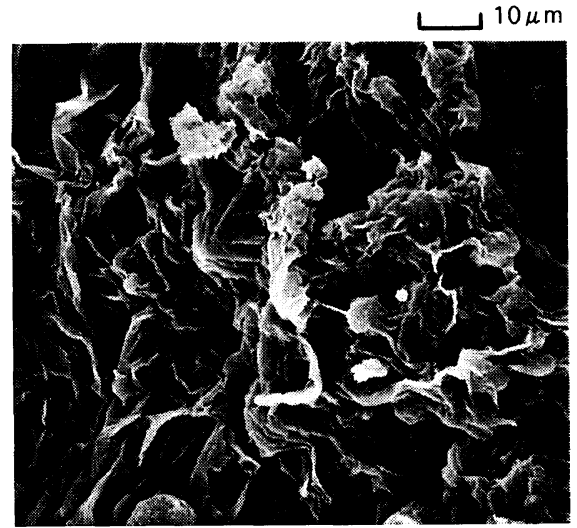

(b) $\mathrm{PE} / \mathrm{CB}=1 / 5$

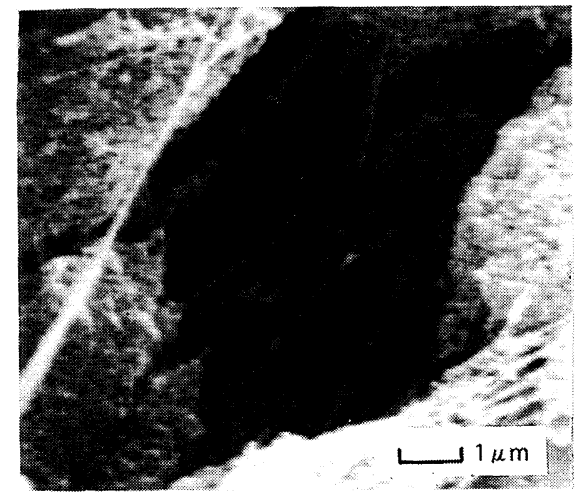

(d) $\mathrm{PE} / \mathrm{CB}=1 / 9$

Figure 5. Scanning electron micrographs for the $1 / 1,1 / 5$, and $1 / 9$ blends. Micrographs (a), (b), and (c) were taken by the same magnification.

observed for the $1 / 1$ blend but any peak shift could not be observed. The drastic decrease in diffraction peak intensity is attributed only to the different crystallinities between PE dry gel film and melt films. Actually, the former and latter crystallinities estimated by density measurements were 84.0 and $57.3 \%$, respectively. For the $1 / 9$ blend, however, the difference between the two $\mathrm{X}$-ray profiles is not significant (see column (b)) because of small amounts of PE content within the blend.

Figure 4 shows SAXS and WAXD patterns (end view) from the blends with various UHMWPE-L.S. CB compositions. As can be seen in the patterns of the dry gel PE film (1/0 composition) without CB particles, the WAXD pattern indicates preferential orientation of the $c$-axes perpendicular to the film surface and the corresponding SAXS pattern, the meridional scattering maxima corresponding to a long period of about $11 \mathrm{~nm}$. The two patterns indicate that within the lamellar crystals constituting the gel, the $c$-axes are oriented perpendicular to the large flat faces in a manner similar to mats of single crystals. ${ }^{36}$ However, the introduction of $20 \%$ CB particles ( $1 / 0.25$ composition) causes the drastic orientational fluctuation of crystal lamellae. It should be noted that the apparent densities of PE and L.S. CB particles are 0.976 and $0.336 \mathrm{~g} \mathrm{~cm}^{-3}$, respectively, since the CB particles contain a number of voids. Actually, $1 / 0.25$ composition by a weight fraction corresponds to $1 / 0.726$ by the volume fraction. The introduction of such amounts of $\mathrm{CB}$ particles hampers the orientation of the constituent lamellar crystal parallel to the material surface, when the as-cast PE gels are dried by slow evaporation of the solvent. The WAXD pattern shows diffraction rings from the (110) and (200) planes and SAXS pattern, circular-type diffuse scattering. As the content of $\mathrm{CB}$ particles increases, the diffuse scattered intensity from CB particles becomes greater in the SAXS pattern and the pattern becomes more indistinct.

Figure 5 displays scanning electron micrographs for the $1 / 1,1 / 5$, and $1 / 9$ blends. For the $1 / 1$ blend, L.S. CB particles were not observed on the surface and only the PE fibrillar texture was observed. This is thought to be due to the fact that the surface free energy of $C B$ is much higher than that of PE. The same was also observed for the $1 / 5$ blend. In micrograph (c), however, a number of CB particles within the $1 / 9$ blend were observed on the film surface because of very high content of CB particles such as $90 \%$. The enlarged micrograph (d) illustrates a micro-crack and a number of continuous fine filaments across the crack. The fine filaments enter-twine the CB particles and the $C B$ particles were fixed in the $1 / 9$ blend material. Actually, the CB particles were not fallen away by weak frictional force on the surface. Interestingly, the block with $40 \mathrm{~mm} \times 20 \mathrm{~mm} \times 20 \mathrm{~mm}$, which was prepared only to estimate the impact resistance, was not broken when the block at $3 \mathrm{~m}$ high was fallen plumb down to a concrete floor. This indicates that the impact resistance of the $1 / 9$ blend is enough to use as the industrial materials.

Columns (a) and (b) in Figure 6 show the change in the profile of the DSC curves of the original and melt specimens for $1 / 0,1 / 0.25,1 / 1,1 / 3,1 / 5$, and $1 / 9$ compositions. The melt specimens are the materials prepared by heat-treatment of the original materials at $180^{\circ} \mathrm{C}$ for $22 \mathrm{~min}$ and recrystallized them at room temperature as 


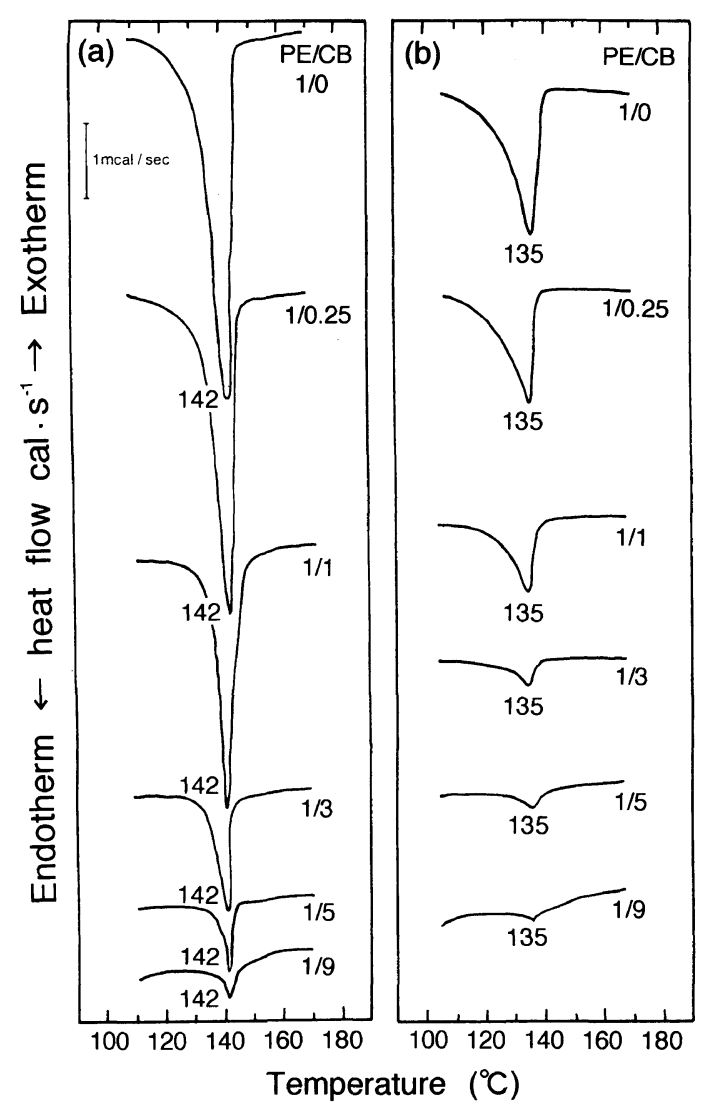

Figure 6. DSC curves of the original and melt specimens with the indicated compositions.

(a) original specimens; (b) melt specimens.

discussed before. To check the reproducibility of the profiles, DSC measurements for a given composition were carried out several times. On columns (a) and (b), DSC curves exhibit a sharp peak for the dry gel PE homopolymer film (1/0) and the corresponding peak of the melt film becomes duller by the heat-treatment at $180^{\circ} \mathrm{C}$. The melting point of the dry gel films appears around $142^{\circ} \mathrm{C}$ but it shifts to $135^{\circ} \mathrm{C}$ by the heat-treatment. This is due to a drastic decrease in crystallinity from 84.0 to $57.3 \%$ as well as the decrease in crystal size. This supports the drastic decrease in peak intensity of the (110) and (200) reflections shown in Figure 3. As can be seen in a series of DSC curves of the blends, increase in $\mathrm{CB}$ content causes significant effect on the peak profile. The peak becomes smaller in proportional to the CB content but the peak position is hardly affected by the $\mathrm{CB}$ content, indicating that the crystallinity and crystal size of PE dry gels within the material are almost independent of the introduction of the CB particles. The melting point for all the melt specimens appears at $135^{\circ} \mathrm{C}$ corresponding to the melting point of PE melt films and the peak of each DSC curve for the melt specimens becomes smaller with increasing CB content. This suggests that the crystallinity and crystal size of $\mathrm{PE}$ by the heat-treatment are independent of the content of $\mathrm{CB}$ particles and then the characteristics of UHMWPE within the material are hardly affected by the introduction of CB particles. This is in good agreement with the variation of the (110) and (200) reflections shown in Figure 3.

Figure 7 shows electric conductivity against content

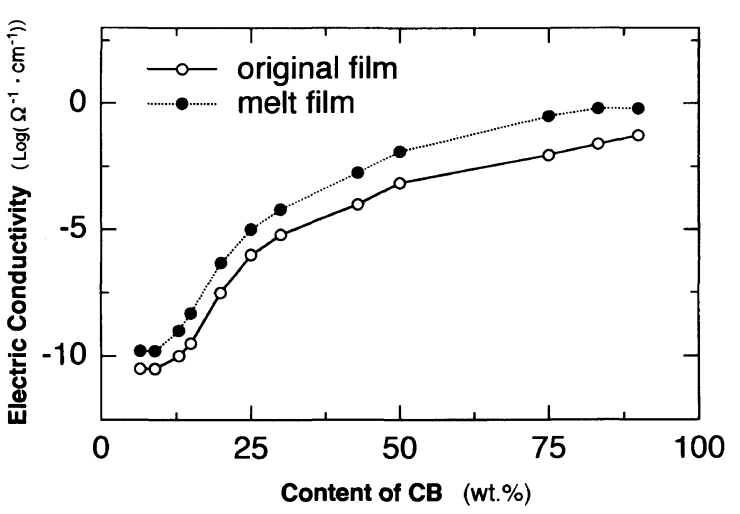

Figure 7. Electrical conductivity measured for original and melt films as a function of $\mathrm{CB}$ content.

of L.S. CB particles. As discussed in ExPERIMENTAL section, electric conductivity was measured at a constant current or a constant voltage using the two-terminal clamping method. Electric conductivity increased with L.S. CB content. The value in Figure 7 reached $1 \Omega^{-1} \mathrm{~cm}^{-1}$ at $90 \% \mathrm{CB}$ content ( $1 / 9$ composition). The value for the melt materials with $1 / 9$ composition is close to $10 \Omega^{-1} \mathrm{~cm}^{-1}$ corresponding to the intrinsic value of CB. ${ }^{37}$ In the given range of CB content, the values were $10^{-9}-1 \Omega^{-1} \mathrm{~cm}^{-1}$ corresponding to semiconductors. Electric conductivity of the melt materials is higher than that of the original specimens, due to the easy alignment of $\mathrm{CB}$ particles to form conductive channels owing to the disappearance of a number of voids by the heattreatment at $180^{\circ} \mathrm{C}$. The crystallinity could not be estimated directly for the blend materials. But, from the comparison with the X-ray data for the $1 / 1$ blend $(50 \%$ CB content) in Figure 3(a), it is evident that the decrease in crystallinity also played a part in increasing conductivity. The electric conductivity, $1 \Omega^{-1} \mathrm{~cm}^{-1}$, for the $1 / 9$ blend ( $90 \%$ CB content) has never been reported for any $\mathrm{PE}-\mathrm{CB}$ blend system. This is due to the fact that although the common preparation of LMWPE-CB blends by kneading has been usually used, this method cannot prepare blends with $\mathrm{CB}$ content $\geqq 65 \%$.

In the given range of $\mathrm{CB}$ content, the conductivity depends on the temperature of heat-treatment of original materials. According to the preliminary experiments, the conductivity of blend for each CB content increased with temperature of heat-treatment of the original film but took a constant value, when the temperature was beyond $220^{\circ} \mathrm{C}$. Incidentally, conductivity of the specimen heattreated at temperature $>190-200^{\circ} \mathrm{C}$ was slightly higher than those of melt films shown in Figure 7. Even so, $180^{\circ} \mathrm{C}$ was selected as the heat-treatment temperature in the present experiments. $180^{\circ} \mathrm{C}$ was the lowest temperature to vanish a number of voids within the specimen without harming the original morphology of PE dry gels. The residual trace of the morphology was confirmed by SAXS and WAXD patterns.

Figure 8 shows temperature dependence of the electric conductivity for melt films with various $\mathrm{CB}$ contents. The measurements were done by the two-terminal clamping method. As can be seen in this figure, the conductivities of all the specimens are almost independent of temperature up to $175^{\circ} \mathrm{C}$ indicating thermal stability. Such thermal stability beyond the equilibrium melting 


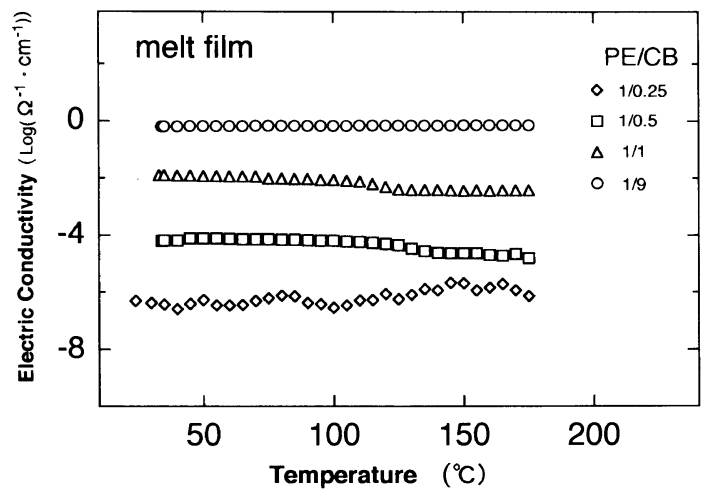

Figure 8. Temperature-dependence of electrical conductivity measured for melt films with various CB contents.

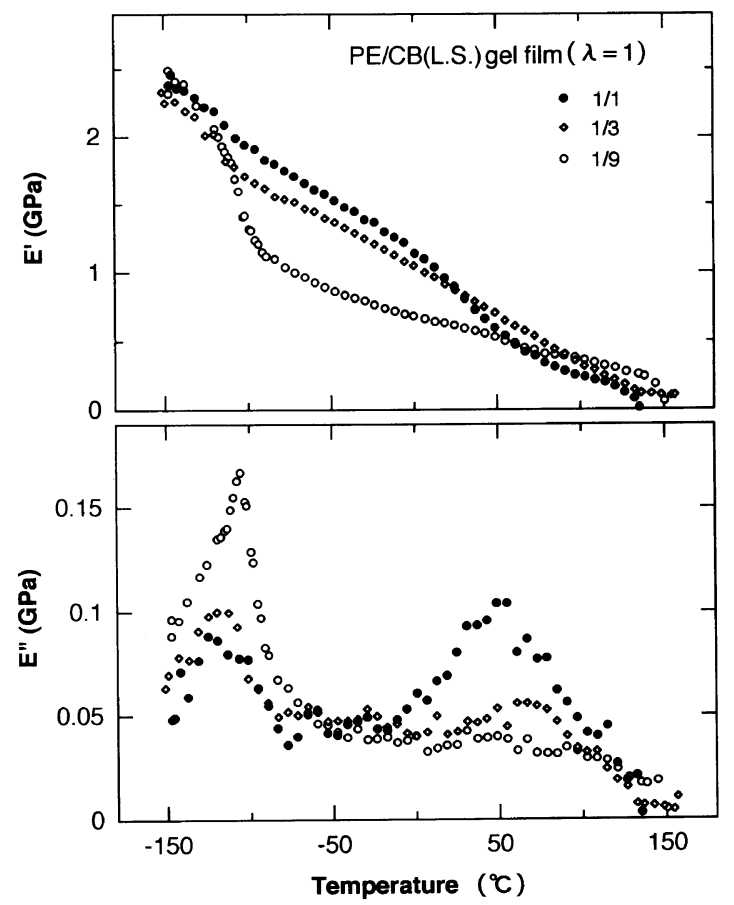

Figure 9. Temperature dependence of the storage and loss moduli measured for PE-L.S. CB films with $1 / 1,1 / 3$, and $1 / 9$ compositions.

point $\left(145.5^{\circ} \mathrm{C}\right)$ of $\mathrm{PE}^{27}$ is thought to be due to two factors; 1) large difference of surface free energy between $\mathrm{PE}$ and $\mathrm{CB}$ and 2) very small melt index value of UHMWPE. The former is due to the fact that CB particles do not appear on the material surface and are maintained in the gaps between PE fibrils. The latter plays an important role to maintain the shape of materials beyond the equilibrium melting point. The drastic decrease in conductivity leading to the volume expansion of UHMWPE occurs at about $135^{\circ} \mathrm{C}$ for the blend with $13 \% \mathrm{CB}$ content, although decrease was much less in comparison with LMWPE-CB blends prepared by kneading. This is well known as the positive temperature coefficient (PTC). This analysis shall be discussed elsewhere. ${ }^{38}$

Figure 9 shows the temperature dependence of the storage and loss moduli for materials with $1 / 1,1 / 3$, and $1 / 9$ compositions. As can be seen in a series of experimental results, the storage modulus decreased with increasing temperature. The value of the $1 / 9$ blend was lowest in the temperature range from -110 to $55^{\circ} \mathrm{C}$.
Table I. Tensile strength and Young's modulus measured for blends at maximum draw ratio

\begin{tabular}{|c|c|c|c|c|}
\hline CB Content & $\begin{array}{c}20 \% \\
(1 / 0.25)\end{array}$ & $\begin{array}{c}30 \% \\
(1 / 0.5)\end{array}$ & $\begin{array}{l}50 \% \\
(1 / 1)\end{array}$ & $\begin{array}{l}75 \% \\
(1 / 3)\end{array}$ \\
\hline Maximum draw ratio & 150 & 110 & 70 & 25 \\
\hline Tensile strength (GPa) & 1.31 & 0.58 & 0.46 & - \\
\hline Young's modulus (GPa) & 73.6 & 37.5 & 25.4 & - \\
\hline
\end{tabular}

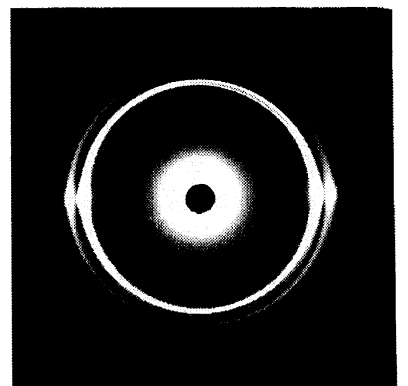

(a) $\lambda=10$

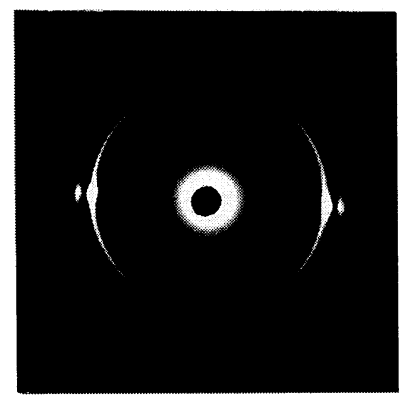

(b) $\lambda=30$

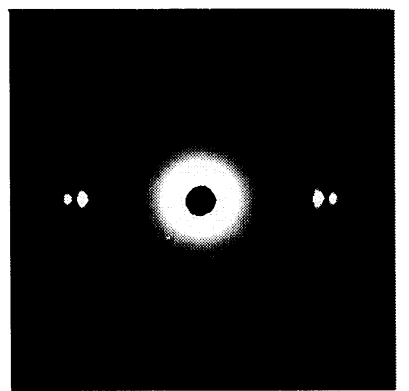

(c) $\lambda=50$

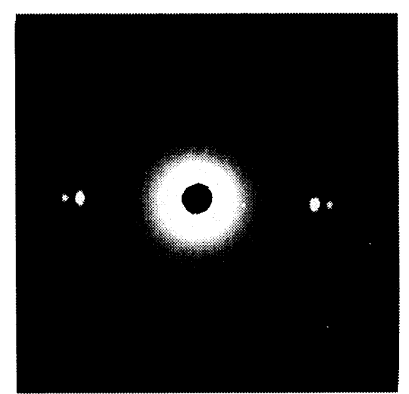

(d) $\lambda=70$
Figure 10. WAXD patterns from the $1 / 1$ blends with the indicated draw ratios.

Beyond $55^{\circ} \mathrm{C}$ associated with the crystal dispersion (the $\alpha$ transition), ${ }^{39,40}$ however, the decrease in storage modulus for the $1 / 9$ blend becomes less in comparison with the other two blends and the value maintains highest at temperature $>90^{\circ} \mathrm{C}$. This is due to the fact that the storage modulus of $\mathrm{CB}$ particles is almost independent of temperature and decrease in the storage modulus tends to modulate in comparison with other two specimens with smaller CB contents. A drastic increase in the storage modulus was confirmed for the $1 / 9$ blend at temperatures $<-100^{\circ} \mathrm{C}$. Such a drastic increase has significant effect on corresponding loss modulus. The dispersion peak, the $\gamma$ transition, appeared at $-110-120^{\circ} \mathrm{C}$, reported as the local motion of side groups associated with the amorphous friction and defects in the crystalline phase. ${ }^{41}$ The peak for the 1/9 blend was a very sharp and is related to the drastic increase in $\mathrm{E}^{\prime}$ at temperature range $<-100^{\circ} \mathrm{C}$. Judging from very small amounts of PE within the $1 / 9$ blend, the appearance of the sharp peak is thought to be due to the friction at boundary regions between CB particles and PE gels. If this was attributed to a number of defects in the crystalline phase which came out by the introduction of large amounts of $C B$ particles, the melting point of the UHMWPE dry gels within the $1 / 9$ blend must shift to lower temperature drastically. The DSC peak was maintained at $142^{\circ} \mathrm{C}$ corresponding to the melting point of the UHMWPE dry gel films as shown in Figure 6. A very small broad 
peak of the $\beta$ transition associated with amorphous dispersion was observed for all specimens at -50 $-20^{\circ} \mathrm{C}$ because of high crystallinity of PE dry gels.

Table I shows the achievable maximum draw ratio $\lambda_{\max }$ of the composite materials with UHMWPE-CB compositions and corresponding tensile strength and Young's modulus. The draw ratio decreased as CB content increased and consequently the tensile strength and Young's modulus decreased. The tensile strength and Young's modulus of the $1 / 0.25$ blend with $\lambda_{\max }=150$ reached 1.31 and $73.6 \mathrm{GPa}$, respectively and those of the $1 / 1$ blend with $\lambda_{\max }=70$ reached 0.46 and $25.4 \mathrm{GPa}$, respectively. These mechanical values are enough as industrial materials. The draw ratio of the $1 / 3$ blend reached 25-fold but the tensile strength and Young's modulus were too scattered to list in Table I. For the materials with $\mathrm{CB}$ content $>83 \%$, elongation was impossible in spite of good impact resistance.

Drawn Composite Materials

Figure 10 shows change in WAXD patterns from the
$1 / 1$ blends with increasing draw ratio $\lambda$. The blend materials were prepared using L.S. CB particles. As shown in pattern (a) at $\lambda=10$, the reflections from (110) and (200) planes indicate preferential orientation of the $c$-axes associated with equatorial reflection arcs and random orientation associated with diffraction rings. At $\lambda=30$, a similar orientational mode was confirmed from the strong equatorial spots and large reflection arcs. The patterns (c) at $\lambda=50$ and the pattern (d) at $\lambda=70$ show strong equatorial spots, indicating a high degree of orientation of the $c$-axes. Of course, it is evident that the orientation is more pronounced with increasing draw ratio. Such drastic increase in orientation of the $c$-axes has significant effect on the tensile strength and Young's modulus as listed in Table I. Incidentally, the diffuse scattering halo from $\mathrm{CB}$ particles was too weak to observe for any specimen because of a drastic decrease in the number of $\mathrm{CB}$ particles within the X-ray irradiated volume by elongation beyond $\lambda=10$.

Figure 11 shows scanning electron micrographs for the $1 / 1$ blend with a draw ratio of 50 ensuring high

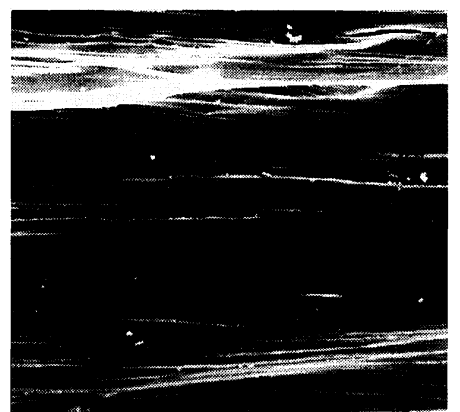

(a) L.S.

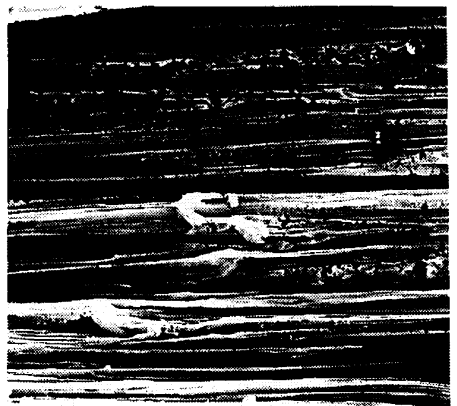

(b) H.S.

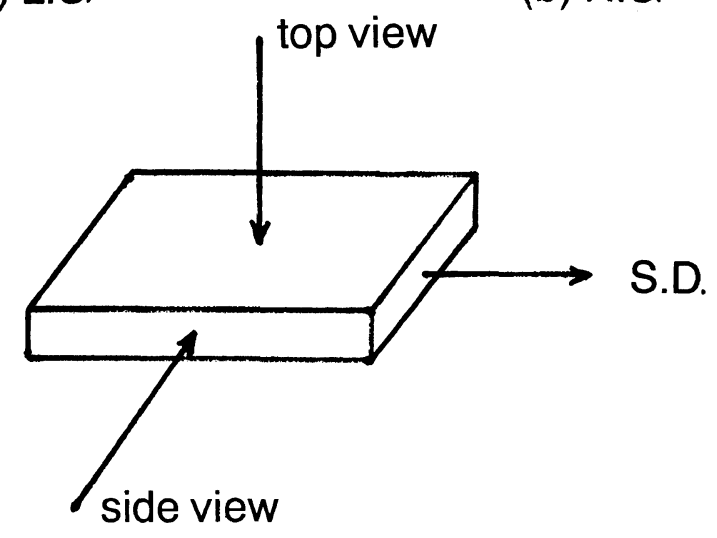

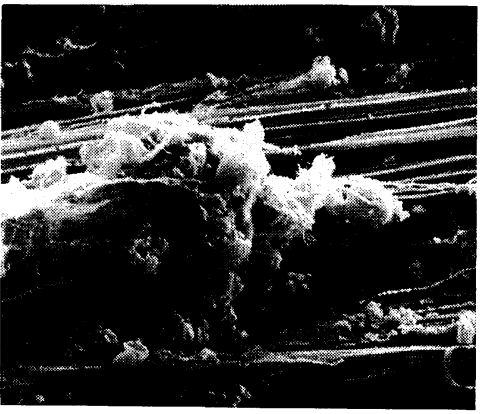

(c) L.S.

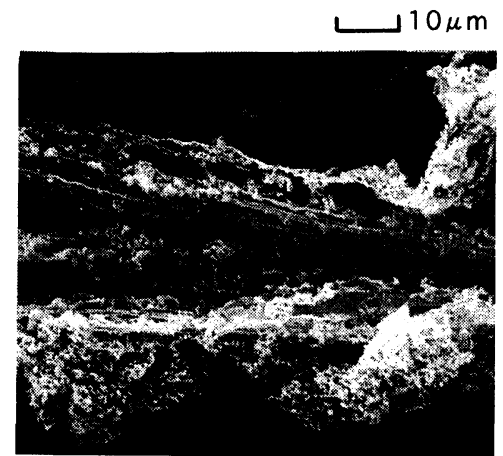

(d) H.S.

Figure 11. Scanning electron micrographs for the blends with UHMWPE-L.S. CB $=1 / 1$ and UHMWPE-H.S. CB $=1 / 1$. Draw ratio is 50 fold. All the micrographs were taken by the same magnification, in which (a) and (b) are top view and (c) and (d) are side (end) view. 


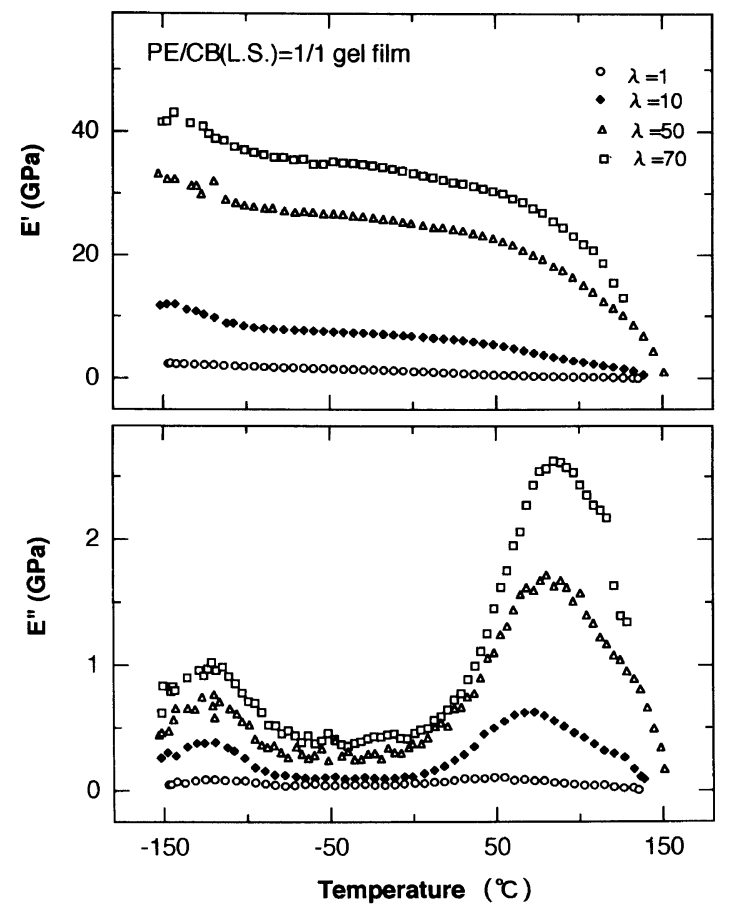

Figure 12. Temperature-dependence of the storage and loss moduli for the $1 / 1$ blends with the indicated draw ratios.

orientation of the $c$-axes (see the WAXD pattern (c) in Figure 10). Because of very high surface free energy of the CB, the L.S. and H.S. CB particles could not be observed on the surface of the drawn material and the highly oriented fibrils could be observed as shown in micrographs (a) and (b) (top view). In the micrographs (c) and (d) (side view), most of the CB particles are pocketed in the large gap between highly oriented fibrils and this tendency is significant for the L.S. CB particles. Under elongation, no CB particle which fell drop from the material could be observed by the naked eye. It is very difficult to check whether the CB particles can maintain continuous tissue such as beads-like-structure without cutting off under sample elongation.

Figure 12 shows the temperature dependence of the storage and loss moduli for the $1 / 1$ blends drawn up to $\lambda=70$. The storage modulus increases with $\lambda$ because of increase in orientation of PE molecular chains (see Figure 10). Because of high crystallinity of the drawn specimens, the $\beta$ transition peak could not be observed for the drawn systems but the $\gamma$ transition peak becomes sharper as the draw ratio increases.

CB particles were maintained within the drawn blends during the elongation (see Figure 11). This is very important for preparing high modulus UHMWPE-CB blends. Actually, the storage moduli of the specimen with $\lambda=70$ reached $34 \mathrm{GPa}$ at $20^{\circ} \mathrm{C}$. This value is slightly higher than the Young's modulus $(25.4 \mathrm{GPa})$ estimated by a stress-strain curve (see Table I).

Figure 13 shows the anisotropy of electric conductivity for the stretching and film thickness directions for the $1 / 1$ blend as a function of the draw ratio. For UHMWPE-L.S. CB blends, conductivity in the stretching direction decreased gradually. In contrast, electric conductivity in the thickness direction increased slightly. The same was observed for UHMWPE-H.S. CB blends. At the indicated draw ratios, conductivity for

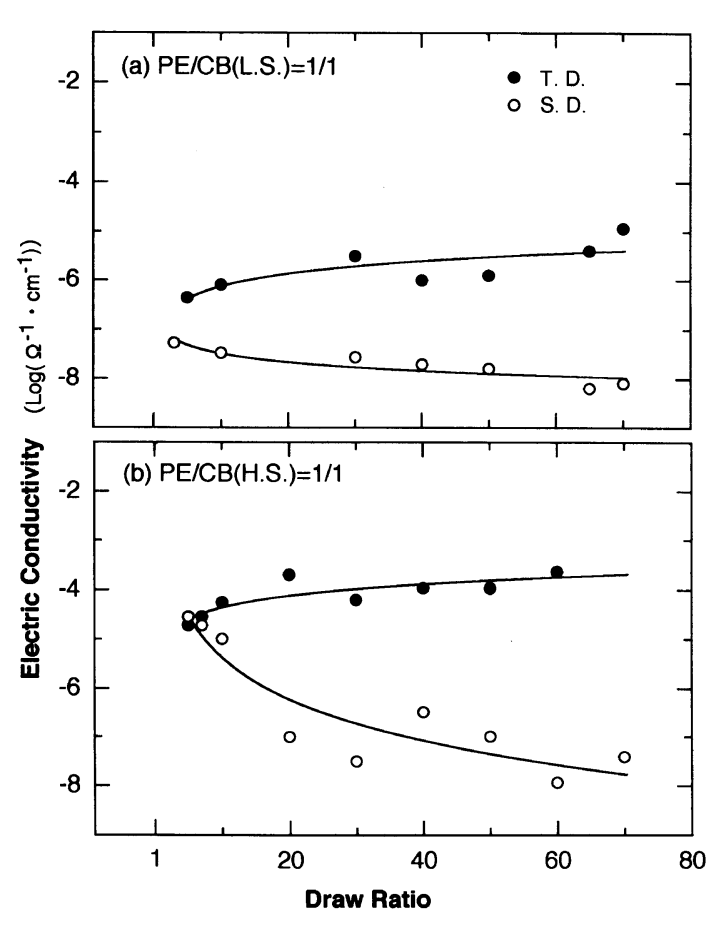

Figure 13. Electrical anisotropy as a function of draw ratio in the stretching and film thickness directions. Measurements were done for blends with $1 / 1$ composition.

UHMWPE-H.S. CB blends was higher than for UHMWPE-L.S. CB blends in the film thickness direction. Conductivity in the stretching direction decreased drastically with increasing draw ratio and reached $10^{-8} \Omega^{-1} \mathrm{~cm}^{-1}$, close to that for L.S. CB particles. The decrease in electric conductivity in the stretching direction is thought to be due to less possibility of jointed CB particles on unit surface area of a drawn specimen, since the specimen did not deform at constant volume. This is related to the characteristic sponge-like-structure of specimens prepared by gelation/crystallization from solutions. Specimens contain many voids and large PE lamellae and the decrease in width of the specimen is much smaller than that in length (corresponding to the stretching direction) by high elongation. ${ }^{36,42}$ Slight increase in the thickness direction indicates retention of the continuous tissue of $\mathrm{CB}$ particles and further contact between $\mathrm{CB}$ particles by planner compression under elongation. Anyway, the best condition to ensure the continuous tissue of $\mathrm{CB}$ particles must be taken into account in further studies to prepare high modulus and high strength semi-conductive materials.

\section{CONCLUSION}

Composite materials were prepared by the introduction of CB particles into UHMWPE solutions and subsequently by the gelation/crystallization from the solutions. UHMWPE-CB compositions chosen were $1 / 0.25,1 / 0.5,1 / 0.75,1 / 1,1 / 3,1 / 5$, and $1 / 9$. The materials provided the UHMWPE-CB blended system with $90 \%$ $\mathrm{CB}$ content and electric conductivity was $10^{-9}-1 \Omega^{-1}$ $\mathrm{cm}^{-1}$, corresponding to semiconductors. These values were almost independent of temperature up to $175^{\circ} \mathrm{C}$ indicating thermal stability at $\mathrm{CB}$ content $>25 \%$. Drawability was less pronounced as CB content in- 
creased. Namely, the maximum achievable draw ratio of the blend with $1 / 1$ composition $(50 \% \mathrm{CB}$ content) was 70 -fold, while that with $1 / 0.25$ composition $(20 \% \mathrm{CB}$ content), 150-fold. The corresponding Young's modulus and tensile strength were 25.4 and $0.46 \mathrm{GPa}$, respectively, for the $1 / 1$ blend, while 73.6 and $1.31 \mathrm{GPa}$, respectively for the $1 / 0.25$ blend. The electric conductivity in the stretching direction decreased with increasing draw ratio, while in the thickness direction slightly increased.

\section{REFERENCES}

1. H. Scher and R. Zallen, J. Chem. Phys., 53, 3759 (1970).

2. B. Buche, J. Appl. Phys., 43, 4837 (1972).

3. F. Baeche, J. Appl.Phys., 44, 532 (1973).

4. J. Meyer, Polym. Eng. Sci., 13, 462 (1973).

5. J. Meyer, Polym. Eng. Sci., 14, 706 (1974).

6. C. Klason and J. Kubat, J. Appl. Polym. Sci., 19, 831 (1975).

7. M. Narkis, A. Ram, and Z. Stain, Polym. Eng. Sci., 21, 1049 (1981).

8. K. T. Chung, A. Sabo, and A. P. Pica, J. Appl. Phys., 53, 6867 (1982).

9. K. Miyasaka, K. Watanabe, E. Jojima, H. Aida, M. Sumita, and K. Ishikawa, J. Mater. Sci., 17, 1610 (1982).

10. M. Sumita, S. Absai, N. Miyadera, E. Jojima, and K. Miyasaka, Colloid Polym. Sci., 274, 212 (1986).

11. M. Sumita, H. Abe, H. Kayaki, and J. Miyasaka, Macromol. Sci., Phys., B25, 171 (1986).

12. R. D. Sherman, L. M. Middleman, and S. M. Jacobs, Polym. Eng. Sci., 23, 36 (1983).

13. D. Malachlam, J. Phys., C, 20, 865 (1987).

14. K. Yoshida, Y. Tomii, and S. Ueda, Jpn. J. Appl. Phys., 27, 2224 (1988).

15. S. Asai, M. Hayakawa, M. Suzuki, M. Sumita, K. Miyasaka, and H. Nakagawa, Kobunshi Ronbunshu, 48, 635 (1991).

16. M. Blaszkiewicz, D. S. Mclachlan, and R. E. Newnham, Polym. Eng. Sci., 32, 421 (1992)
17. F. Lux, Polym. Eng. Sci., 33, 334 (1993)

18. H. Tang, J. Piao, X. Chen, Y. Luo, and S. Li, J. Appl. Polym. Sci., 48, 1795 (1993).

19. W. Jia and X. Chen, J. Appl. Polym. Sci., 54, 1219 (1994).

20. Y. Agari, A. Ueda, and S. Nagai, J. Appl. Polym. Sci., 52, 1223 (1994).

21. L. Yang and D. L. Schruben, Polym. Eng. Sci., 34, 1109 (1994)

22. L. Karasek and M. Sumita, J. Mater. Sci., 31, 281 (1996).

23. N. Tsubokawa, A. Funaki, Y. Hada, and Y. Sone, J. Polym. Sci., Polym. Chem. Ed., 20, 3297 (1982).

24. N. Tsubokawa, Gomu Kyokai 58, 306 (1985)

25. K. Ohe and Y. Naito, Jpn. J. Appl. Phys., 10, 99 (1971).

26. T. Kimura, Y. Asano, and S. Yasuda, Polymer, 37, 2981 (1996)

27. P. J. Flory and A. J. Vrij, J. Am. Chem. Soc., 85, 3458 (1963).

28. P. Smith, P. J. Lemstra, B. Kalb, and A. J. Pennings, Polym. Bull., 1, 733 (1979).

29. P. Smith, and P. J. Lemstra, J. Mater. Sci., 15, 505 (1980).

30. P. Smith, P. J. Lemstra, J. P. L. Pippers, and A. M. Kiel, Colloid Polym. Sci., 258, 1070 (1981).

31. M. Matsuo, K. Inoue, and N. Abumiya, Sen'i Gakkaishi, 40, 275 (1984).

32. M. Matsuo, C. Sawatari, M. Iida, and M. Yoneda, Polym. J., 17, 1197 (1985).

33. M. Matsuo, and C. Sawatari, Macromolecules, 19, 2036 (1986).

34. C. W. Bunn, Trans. Faraday Soc., 35, 482 (1939).

35. M. Matsuo, C. Sawatari, and T. Ohhata, Macromolecules, 21, 1317 (1988).

36. M. Matsuo and R. S. J. Manley, Macromolecules, 15, 7985 (1982).

37. W. D. Schaeffer, W. R. Smith, and M. H. ZPolley, Ind. Eng. Chem., 45, 1721 (1953).

38. M. Matsuo, in preparation.

39. H. Nakayasu, H. Markovitz, and D. J. Plazek, Trans. Soc. Rheol., 5, 261 (1961).

40. M. Takayanagi and M. Matsuo, J. Macromol. Sci., Phys., B1, 407 (1967).

41. J. D. Hoffman, G. Williams, and E. Oassaglia, J. Polym. Sci., Part C, 14, 173 (1966).

42. M. Matsuo and R. S. T. Manley, Macromolecules, 16, 1500 (1983). 